\title{
Assessment of the relative sensitivity of milk ELISA for detection of Mycobacterium avium ssp. paratuberculosis infectious dairy cows
}

\author{
Emilie L. Laurin, ${ }^{1}$ Javier Sanchez, Marcelo Chaffer, Shawn L. B. McKenna, and Greg P. Keefe \\ Department of Health Management, Atlantic Veterinary College, University of Prince Edward Island, 550 University Avenue, Charlottetown, \\ Prince Edward Island C1A 4P3, Canada
}

\begin{abstract}
Milk ELISA are commonly used for detection of Mycobacterium avium ssp. paratuberculosis (MAP) antibodies in dairy cows, due to low cost and quick processing for large numbers of samples. However, low sensitivity and variations from host and environmental factors can impede detection of MAP antibodies at early disease stages. The objectives of our study were to assess the sensitivity of milk ELISA in comparison with fecal tests and to evaluate how detectable antibody concentrations in milk vary with changes in fecal shedding of MAP, cow age, cow parity, days in milk, and time of year. To compare the sensitivity of a commercial milk ELISA with solid and broth fecal culture and with fecal real-time PCR, a longitudinal study was performed for the identification of MAP-infectious animals as determined by prior fecal testing for MAP shedding. In addition, associations between variation in milk MAP ELISA score and changes in fecal MAP shedding, host age, days in milk, and season were evaluated. Monthly milk and fecal samples were collected over $1 \mathrm{yr}$ from 46 cows that were previously shedding MAP in their feces. Sensitivity of milk ELISA was 29.9\% (95\% CI: 24.8 to $35.1 \%$ ), compared with $46.7 \%$ (40.7 to $52.7 \%$ ) for fecal solid culture, $55.0 \%$ (49.3 to $60.7 \%$ ) for fecal broth culture, and $78.4 \%$ (73.3 to $83.1 \%$ ) for fecal direct realtime PCR. The effect of stage of lactation could not be separated from the effect of season, with increased milk ELISA scores at greater days in milk in winter. However, unpredictable monthly variations in results were observed among the 3 assays for individual cow testing, which highlights the importance of identifying patterns in pathogen and antibody detection over time in MAP-positive herds.
\end{abstract}

Key words: milk ELISA, Johne's disease, Mycobacterium avium ssp. paratuberculosis, season, diagnostic sensitivity

Received March 20, 2016.

Accepted September 25, 2016.

${ }^{1}$ Corresponding author: elaurin@upei.ca

\section{INTRODUCTION}

Johne's disease, a production-limiting disease of dairy cattle caused by the organism Mycobacterium avium ssp. paratuberculosis (MAP), can have a substantial financial effect on dairy farmers (Tiwari et al., 2006). Nielsen and Toft (2008) have described 3 groups of MAP-positive cows: MAP-infected cows carrying the organism, MAP-infectious cows shedding detectable amounts of the organism, and MAP-affected cows with clinical signs of the disease. The MAP-infected cows can progress to MAP-infectious stages, often showing intermittent bacterial shedding in feces initially, before becoming clinically MAP affected (Nielsen and Toft, 2008). Some cows in the MAP-infected stage have an antibody response (before fecal shedding and clinical signs) that can be detected via an ELISA. Although occasionally some MAP-infectious cows may not have detectable antibodies, ELISA sensitivity (Se) tends to improve with increased bacterial shedding and clinical stages of disease (Carpenter et al., 2004; Nielsen and Toft, 2006; Tiwari et al., 2006). The presence of antibodies can, therefore, be predictive of an increased risk for fecal MAP shedding (Nielsen, 2008; Lavers et al., 2014). The ELISA Se has also been reported to improve as the age and parity of MAP-positive cows increase, coinciding with increased MAP shedding and clinical signs (Toft et al., 2005; Nielsen et al., 2013).

Adequate diagnostics are essential for management of infected and infectious animals, which is a key component for controlling Johne's disease on a farm (Garry, 2011). Due to imperfect tests sometimes showing variable results and the intermittent bacterial shedding in subclinical Johne's disease (Eisenberg et al., 2015; Lavers et al., 2015), the identification of any antibody detection pattern related to host factors (age, parity, lactation stage) or environmental factors (season) will be beneficial to the dairy industry when using diagnostic assays. The ELISA is more time efficient and cost effective than culture methods, but Se of ELISA is generally poor (29 to $61 \%$ in 5 studies of infectious cows; $39 \%$ in one study of infected cows), with imperfect specificity (Sp; 83 to $98 \%$ in 4 studies of infectious 
cows; 96 to $100 \%$ in 2 studies of infected cows; Nielsen and Toft, 2008). Sensitivity can vary greatly, depending on reference standard choice and its Se and Sp, prevalence of MAP in a herd, and ELISA kit and methodology (Lombard et al., 2006; Lavers et al., 2015). Nielsen and Toft (2006) also addressed the importance of relating Se and Sp to the purpose of testing, and knowing whether infected, infectious, or affected cows are targeted for diagnosis.

Identification of any patterns in MAP pathogen and antibody detection over time in MAP-positive herds can help implement effective management programs that increase producer participation. Because ELISA are commonly used due to their cost and time benefits, it is necessary to understand how ELISA Se, both at the individual and herd levels, varies across lactation stages relative to Johne's disease progression, in the presence of nonspecific colostral antibodies, with milk dilution effects (Nielsen and Toft, 2012), and with seasonal effects (Cazer et al., 2013).

The objectives of our study of individual cow testing were (1) to assess the total and monthly Se of milk ELISA in comparison with solid and broth fecal culture and with fecal real-time PCR (qPCR) for previously identified MAP-infectious cows; and (2) to evaluate how detectable antibody concentrations in milk vary with changes in fecal shedding of MAP, cow age, cow parity, DIM, and time of year.

\section{MATERIALS AND METHODS}

\section{Farm and Cow Selection}

Holstein dairy herds positive for MAP in New Brunswick (4) and Prince Edward Island (3), Canada, were recruited for this longitudinal study. Herds within the study did not have a seasonal calving pattern, but rather had a relatively equal number of calvings per month, as is typical of the Canadian dairy industry. Apparent within-herd MAP prevalence (based on fecal broth culture) for these farms ( 83 to 490 cows per herd) ranged from 3 to $15 \%$ in the previous year [C. Lavers; Atlantic Veterinary College (AVC), Charlottetown, PE, Canada, personal communication]. For this study, the target condition was MAP infectious as defined by fecal MAP shedding detected by culture methods (Nielsen and Toft, 2008). The case definition was any cow that was MAP infectious at least once during the 1-yr period before the start of this study, as determined from a companion study (Lavers et al., 2013) in which MAPpositive cows were identified using pooled fecal broth culture (pools of 5 cows; Trek ESP culture system II; Trek Diagnostic Systems, Thermo Scientific, Oakwood Village, $\mathrm{OH}$ ) confirmed with acid fast stain and qPCR.
Any positive pools had individual fecal samples further cultured as described in the companion study (Lavers et al., 2013). A total of 46 MAP-infectious cows were identified for milk and fecal sampling for this study. Farmers were not blinded to the MAP status of these cows.

\section{Sample Collection}

The Animal Care Committee (University of Prince Edward Island, Charlottetown, PE, Canada) approved all animal protocols before the start of this study. Milk and fecal samples were collected monthly, during lactation periods, for up to 12 mo from their inclusion in the study and for as long as the cows remained in the herds (study period: July 2010 to December 2011). Samples of feces were collected per rectum by the project personnel, using individual, nonlubricated rectal sleeves. Milk samples were collected, following standard milk collection protocols, either by the project personnel or by the farmers. All samples were frozen at $-80^{\circ} \mathrm{C}$ in the USDA proficiency-tested Maritime Quality Milk Laboratory at AVC.

\section{Laboratory Procedures}

Fecal Solid Culture, Broth Culture, and RealTime PCR. Methods for fecal testing followed those described in Laurin et al. (2015). For solid culture, Herrold's egg-yolk medium (HEYM) slants (Becton, Dickinson, and Company, Sparks, MD), supplemented with mycobactin $\mathrm{J}$, amphotericin $\mathrm{B}$, naladixic acid, and vancomycin, were used. For broth culture, the Trek ESP culture system II (Trek Diagnostic Systems) was used, which records MAP growth as a days-to-positive reading, signaled by detection of decreased pressure in the bottle of medium. For real-time PCR, the Tetracore kit protocol (VetAlert Johne's Real-Time PCR, Tetracore, Rockville, MD) was used, which targets the $h s p X$ gene. Confirmatory acid-fast stain was performed for all broth and solid cultures. Any positive culture or any positive acid-fast stain was further confirmed with qPCR following protocols outlined in the VetAlert Johne's Real-Time PCR kit (Tetracore). Cultures were subsequently classified as positive if qPCR was positive.

Milk ELISA. Procedures followed the recommendations of the commercial Parachek-2 ELISA kit (Prionics AG, Schlieren-Zürich, Switzerland), using the kinetic detection method. The adjusted score results [sample raw optical density (OD) minus average of the 2 negative control OD values] were set at $<0.07$ as negative, 0.07 to 0.1 as suspicious, and $>0.1$ as positive. Any results within the suspicious category were rerun to classify as either positive or negative. If they stayed in 
the 0.07 to 0.1 range, they were classified as negative, as per kit instructions.

\section{Statistical Analysis}

Statistical analyses were done using STATA/IC.12 (StataCorp LP, College Station, TX), Minitab 16.2.2 (Minitab Inc., State College, PA), and MLwiN (Centre for Multilevel Modeling, Bristol, UK) software, using a $P<0.05$ as a cut-off for statistical significance. Relative Se (proportion of observations that were positive for the previously determined MAP-infectious cows, as described above) for ELISA results were recorded along with a $95 \%$ confidence interval, accounting for clustering by cow. Analysis was limited to data from cows $<400$ DIM to stay within a typical lactation length and because data were sparse above this threshold. The Se of ELISA was compared with each Se for the 3 fecal assays (solid and broth cultures and qPCR). Additionally, agreement analyses, using Cohen's kappa and McNemar's exact test (Dohoo et al., 2009), were calculated between dichotomous milk ELISA and fecal results. For solid culture, low shedding related to $<10$ cfu per culture slant, moderate shedding from 10 to 50 cfu, and high shedding $>50 \mathrm{cfu}$ (Crossley et al., 2005). For broth culture, high shedding related to $<20 \mathrm{~d}$ to positive, moderate shedding between 21 and $28 \mathrm{~d}$, and low shedding between 29 and 49 d (Shin et al., 2000, 2001). For qPCR, high shedding related to $<26$ cycle threshold (Ct), moderate shedding between 26 and 30 $\mathrm{Ct}$, and low shedding between 30 and $42 \mathrm{Ct}$, as per previous work (Laurin et al., 2015).

For milk samples, a 2-level (random effects at the cow and farm levels) hierarchical mixed linear regression model (Dohoo et al., 2009) for a continuous ELISA score outcome was used for the analysis as follows:

$$
\begin{aligned}
\mathrm{Y}_{\mathrm{i}}=\text { intercept }+ & \text { season }_{\mathrm{i}}+\mathrm{DIM}_{\mathrm{i}}+\text { age }_{\mathrm{i}} \text { (or parity) } \\
& +\mathrm{u}\left(\mathrm{cow}_{\mathrm{i}}\right)+\varepsilon_{\mathrm{i}},
\end{aligned}
$$

where $\mathrm{Y}$ refers to the continuous ELISA score outcome for cow $\mathrm{i}$, and $\mathrm{u}$ and $\varepsilon$ refer to the random effects. Univariable analysis was explored first, followed by the multivariable model that included the predictors (with $P<0.10$ from the univariable analyses) in a step-wise manner. Analyzed predictors of interest were season, DIM, age, and parity. The collinear age and parity variables were analyzed in separate models. Parity was grouped into first, second, third, fourth, and >fifth parities. Age was categorized into ages 2 and 3, 4, 5, 6, and $>7$ yr. Finally, seasons were categorized into July through September for summer, October through De- cember for fall, January through March for winter, and April through June for spring. Contrasts were analyzed for any predictors with $P<0.05$ and were reported with Bonferroni-adjusted $P$-values for multiple comparisons.

\section{RESULTS}

Infectious cows ranged in ages from 2 to 9 yr (median $=4 \mathrm{yr}$ ) and from first to seventh parity (median = second). From the 46 MAP-infectious cows (range of 1 to 11 observations per cow over the study period), total observations were 304 milk ELISA, 270 fecal solid culture, 298 fecal broth culture, and 297 fecal qPCR. Figures 1, 2, 3, and 4 show any incompatibility and variation in the results of each assay (milk ELISA and fecal assays) for each cow per month over the study period. Of the 46 cows that were previously found to be infectious, only 1 cow had no shedding detected with a fecal pathogen detection method during the study period. This cow was only tested once and then culled. For 14 of the remaining 45 cows with detectable fecal MAP shedding, MAP was detected more than a quarter (range: 25 to $87.8 \%$ ) of their sampling times. The number of samples within ELISA outcomes (positive or negative) was compared with the binary outcomes for the 3 fecal assays in a modified $2 \times 2$ table (Table 1). Kappa between milk ELISA and fecal culture techniques indicated moderate agreement, with 0.57 for solid culture $(P<0.01)$ and 0.46 for broth culture $(P$ $<0.01)$, but poor agreement $(0.19 ; P<0.01)$ was found with fecal direct qPCR. McNemar's test had $P$-values $<0.01$ for all of these assay comparisons for milk and fecal samples.

Relative Se values for milk ELISA, as well as for the 3 fecal diagnostic methods, are listed in Table 2. A higher proportion of positive results for milk ELISA was seen when fecal shedding was greater (Table 3). This trend was significant for high shedding versus low or no shedding detected with fecal solid culture (high versus negative, $P<0.01$; high versus low, $P<$ 0.05 ; high versus moderate, $P=0.09$ ) and qPCR (high versus negative, $P<0.01$; high versus low, $P<0.01$; moderate versus negative, $P=0.06$ ). There was also a trend toward improved Se and greater mean ELISA scores with increasing age. However, after Bonferroni corrections for multiple comparisons, this trend was not significant. A similar trend was also seen with increasing parity (data not shown).

The ELISA scores ranged from -0.17 to 2.42 and were highly positively skewed (mean 0.157, median -0.06 ) and were best transformed to the inverse square root for the regression analysis to meet the assumptions of the linear model. As the predictors for 
age and parity were highly correlated $(\mathrm{r}=0.85, P<$ 0.01 ), both variables were not analyzed in the same model. In the mixed linear regression model, predictors of ELISA score included age $(P<0.05)$, season $(P=$ $0.08)$, and DIM $(P<0.01)$. The model with parity, in place of age, was similar (data not shown). Predicted back-transformed marginal scores (Figure 5) improved toward end of lactation and were more likely to cross the positive threshold $(0.1)$ with increasing age. Scores were higher for winter $(P<0.05)$ than for summer. Numerically, the variation in scores were often quite small and did not necessarily result in an increase in scores above the positive threshold due to few observations in some categories among season, age, and lactation stages.

\section{DISCUSSION}

The reluctance of producers to participate in Johne's control programs may be linked to unreliable and unpredictable test results, due to variation with the commonly used MAP pathogen and antibody detection methods (culture, qPCR, and ELISA) as we observed in our data of monthly sampling (see Figures 1 to 4). Identification of factors influencing Se of milk ELISA for MAP-infectious cows can improve our interpretation of these assays, as part of effective management and control programs.

Our study results are consistent with literature reports of lower milk ELISA Se than standard fecal diagnostics (Tiwari et al., 2006). Nielsen and Toft (2008)

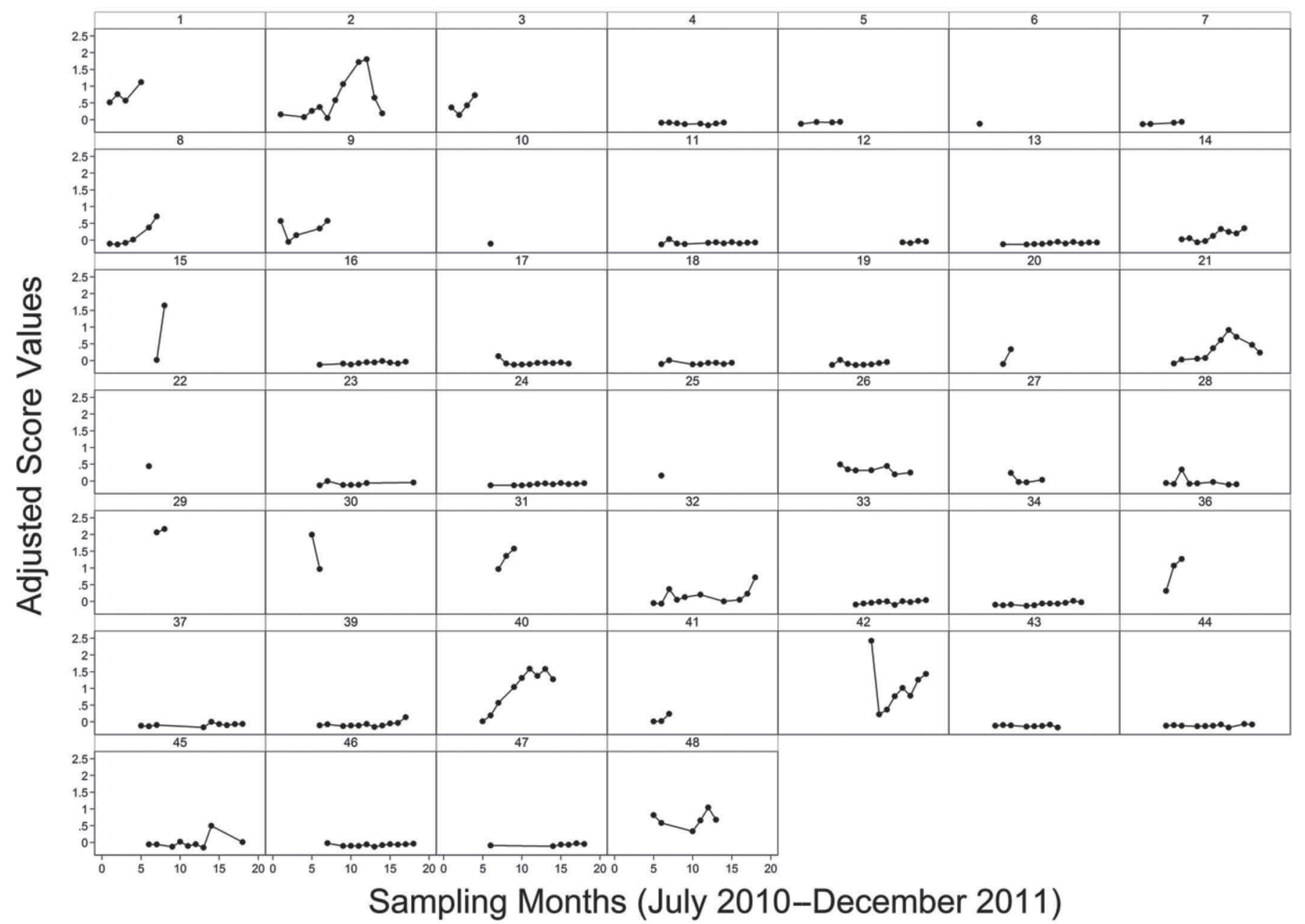

Figure 1. Monthly variation of milk ELISA (Parachek, Prionics, Schlieren-Zürich, Switzerland) adjusted score values for each of the 46 Mycobacterium avium ssp. paratuberculosis infectious dairy cows. Subplots are labelled by the blinded identification number for each cow. The adjusted score results (sample raw optical density minus average of the 2 negative control optical density values) were considered positive at $>0.1$ 


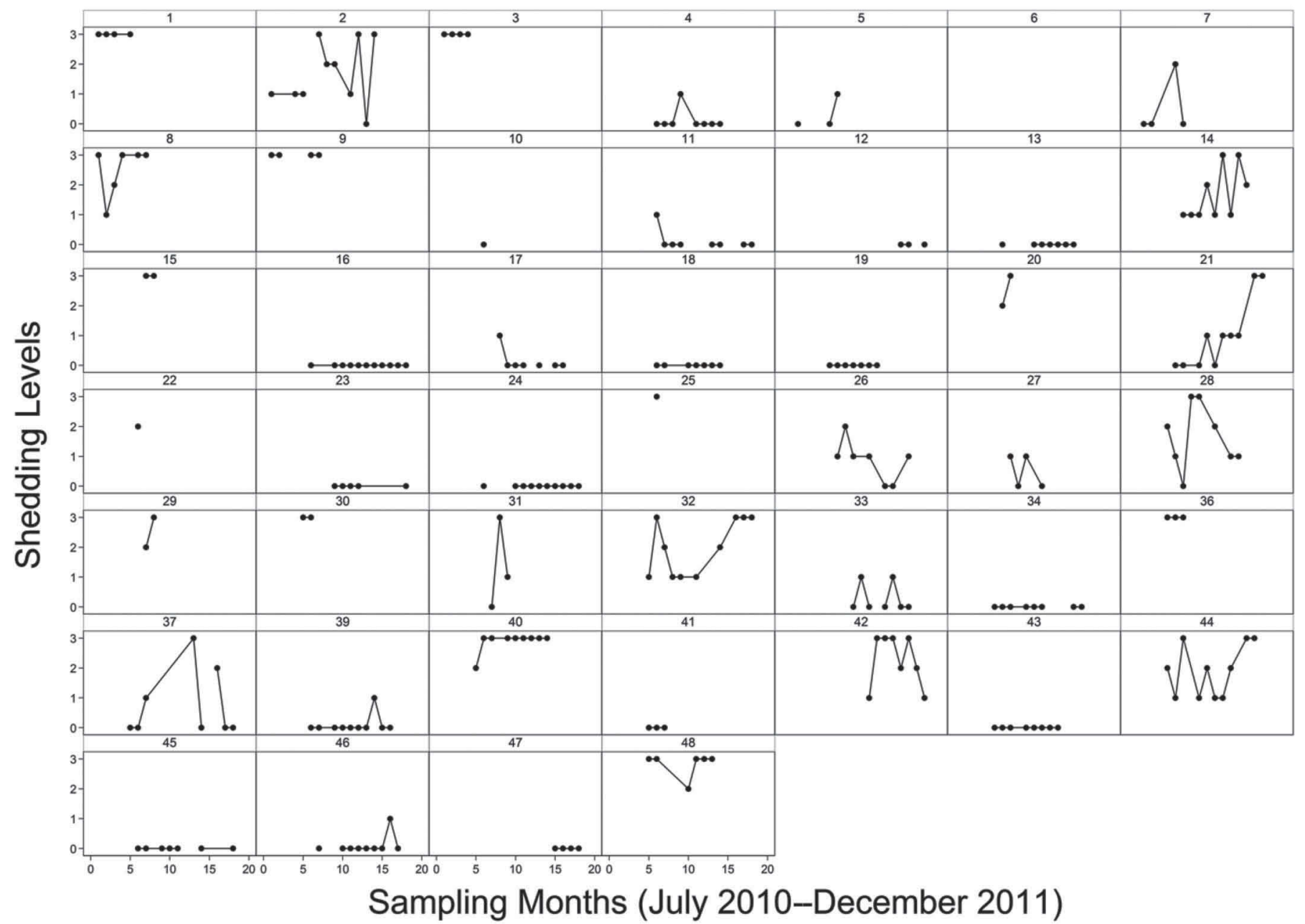

Figure 2. Monthly variation of bacterial growth across shedding levels for fecal solid culture (TREK ESP system, Thermo Scientific, Oakwood Village, $\mathrm{OH}$ ) for each of the 46 Mycobacterium avium ssp. paratuberculosis infectious dairy cows. Subplots are labelled by the blinded identification number for each cow. Shedding categories determined with solid culture: $0=$ no growth, $1=$ low $(<10$ cfu per culture tube), $2=$ moderate (10 to $50 \mathrm{cfu}$ ), and $3=$ high (>50 cfu; Crossley et al., 2005).

assessed the 6 milk antibody ELISA studies done up to that time and found that most studies targeted MAP-infectious cows, with Se ranging from 29 to $61 \%$, whereas a literature review by Tiwari et al. (2006) reported milk Se between 51 and $84 \%$ for subclinical low-shedding cows and subclinical high-shedding cows from high prevalence herds, respectively. Many of these studies used only concurrent high fecal shedding $(>10$ cfu) as a reference standard, as well as sampling from herds with prevalence higher than $25 \%$.

Our study was limited due to the choice of target and case conditions of MAP-infectious cows from the 1-yr period before the study. Milk ELISA results need to be cautiously interpreted relative to the target condition. In our study, only cows that were MAP infectious the previous year were sampled; the negative milk ELISA results would then be interpreted as false negative. It is possible that some of these cows may not have transitioned to a consistently detectable humoral immunity. Generally, ELISA Se should increase with each stage of disease and with increasing numbers of shed bacteria (Carpenter et al., 2004), which we did observe in this study. Nielsen (2008) suggested that cows with repeated positive results on ELISA testing are at higher risk of being MAP infectious and that the ability of cows to become high bacterial shedders could be predicted by the onset of detectable MAP antibodies. Another limitation of our study was the small sample size from farms with a lower prevalence of MAP, which may have resulted in a loss of statistical power in some analyses.

In the current study, moderate agreement was found between milk ELISA and fecal culture tests, and only slight agreement between milk ELISA and fecal qPCR. These findings corresponded with the McNemar's re- 
sults, which indicate that the kappa results should be interpreted with caution. Hendrick et al. (2005) found that, despite a nonsignificant Se difference between an indirect milk ELISA and fecal culture, their level of agreement was greater than that between serum ELISA and fecal culture.

When individual shedding categories for fecal cultures were assessed, our study found a higher proportion of milk ELISA positive results and a $40 \%$ increase in the ELISA score for cows with concurrent heavy MAP shedding ( $>50 \mathrm{cfu}$ on solid culture). Increased Se with increased MAP shedding was similarly observed in a previous study of serum ELISA versus HEYM fecal culture and direct PCR by Clark et al. (2008) and versus HEYM fecal culture by Donat et al. (2014) who also found that the relative Se of serum ELISA was highest at heavy shedding (51-100 cfu) and declined at very high shedding ( $>100 \mathrm{cfu}$ ). Comparisons between studies must be done with careful consideration of the study designs, target conditions, herd prevalence, and ELISA and culture methods (Lombard et al., 2006; Lavers et al., 2015).

Increasing ELISA readings have been previously recorded with increased age and parity (Toft et al., 2005; Nielsen et al., 2013; Donat et al., 2014) especially after 3 yr of age (Huda et al., 2004; Nielsen and Ersbøll, 2006). Interestingly, our study did not detect any positive milk ELISA for 2-yr-old cows, and many results were also negative in 3 -yr-old cows. This pattern may have been a result of study design, as fecal culture positivity within the previous year was used for the case definition. Nielsen and Toft (2006) found milk ELISA Se of $6 \%$ at 2 yr of age and a Se of $50 \%$ at 5 yr of age in MAP-infected cows, but found no change between

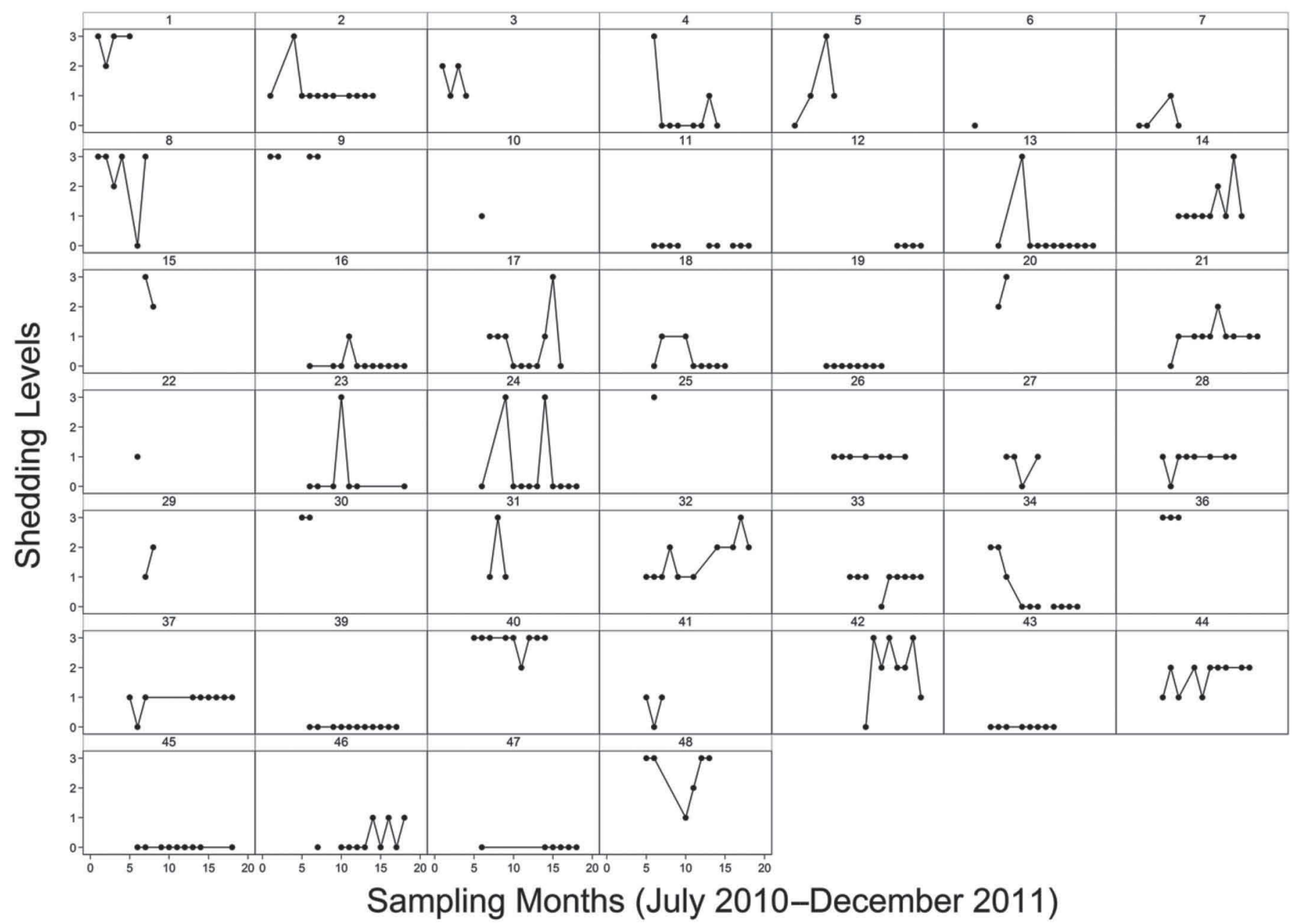

Figure 3. Monthly variation of bacterial growth across shedding levels for fecal broth culture (Herrold's egg yolk medium with mycobactin J) for each of the 46 Mycobacterium avium ssp. paratuberculosis infectious dairy cows. Subplots are labelled by the blinded identification number for each cow. Shedding categories determined with broth culture: $0=$ no growth, $1=$ low ( $>28$ d to a positive signal), $2=$ moderate $(21$ to 28 d), and $3=$ high $(<21$ d; Shin et al., 2000, 2001). 
Table 1. Positive or negative milk ELISA (Paracheck, Prionics, Switzerland) results compared with simultaneous fecal assay results [fecal solid culture (SC; Herrold's egg yolk medium with mycobactin J), fecal broth culture (BC; Trek ESP system, Thermo Scientific, Oakwood Village, $\mathrm{OH}$ ), and fecal real-time PCR (qPCR; VetAlert, Tetracore, Rockville, MD)] for samples collected monthly over a 12-mo period from a total of 46 Mycobacterium avium ssp. paratuberculosis infectious cows from 7 herds ${ }^{1}$

\begin{tabular}{|c|c|c|c|c|c|c|c|}
\hline $\begin{array}{l}\text { Milk } \\
\text { ELISA }\end{array}$ & \multicolumn{3}{|c|}{ Negative } & \multicolumn{3}{|c|}{ Positive } & Total \\
\hline Positive & 9 & 5 & 2 & 78 & 85 & 88 & 87 (SC), 90 (BC), 90 (qPCR) \\
\hline Total & 144 & 134 & 64 & 126 & 164 & 233 & \\
\hline
\end{tabular}

${ }^{1}$ The relative sensitivity of milk ELISA versus concurrent positive fecal SC, BC, and qPCR was $61.9,51.8$, and $37.8 \%$, respectively.

these ages for MAP-infectious cows (Nielsen and Toft, 2006). In comparison, in our study of MAP-infectious cows, just over $30 \%$ Se at age 5 was detected, and Se did not exceed $50 \%$ until $7 \mathrm{yr}$ of age.
The variation in increasing ELISA scores over time recorded in our study was sometimes numerically small and did not necessarily cross the positive threshold. Lavers et al. (2015) reported that ELISA values close

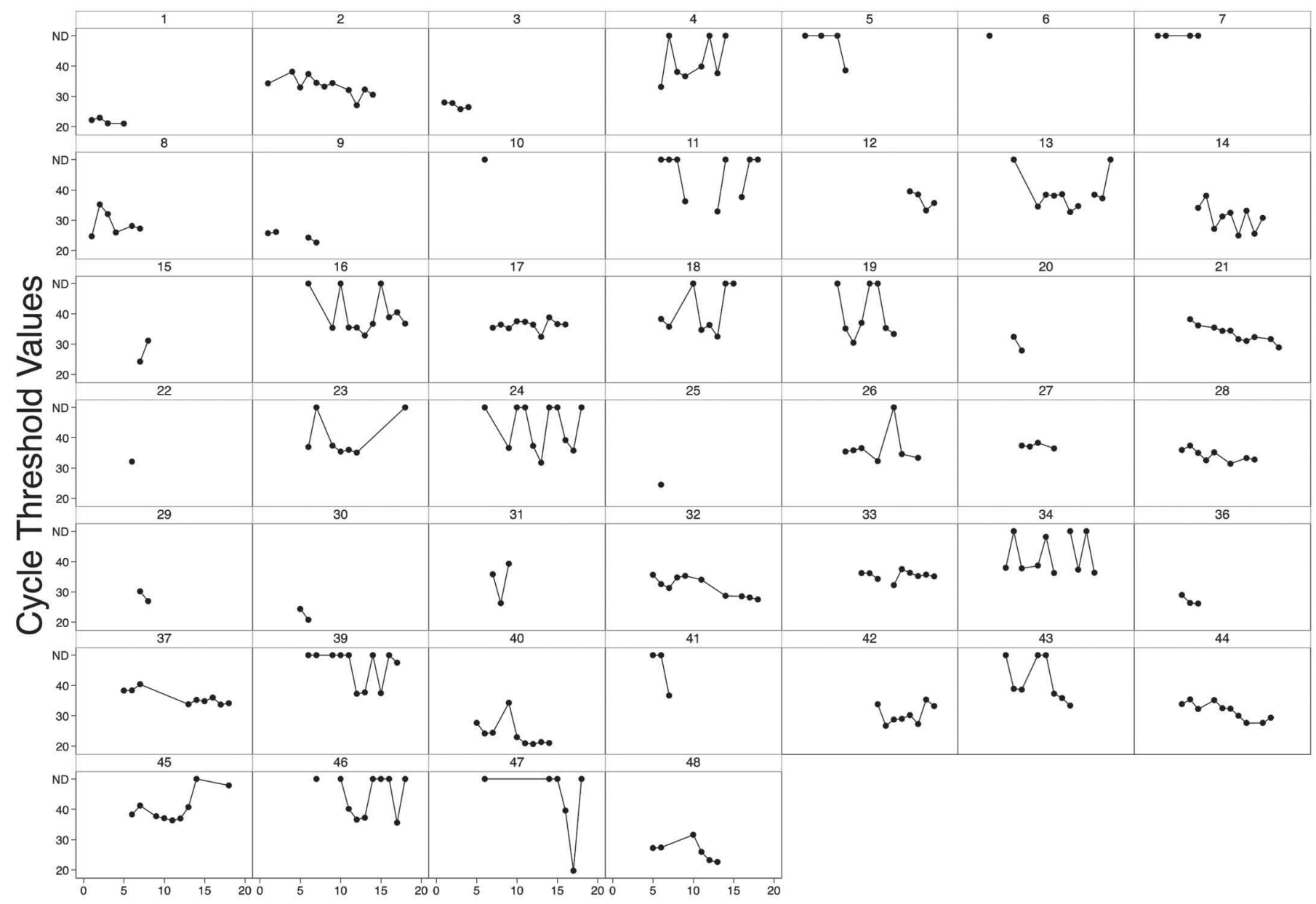

\section{Sampling Months (July 2010-December 2011)}

Figure 4. Monthly variation of cycle threshold values for fecal direct real-time PCR (VetAlert, Tetracore, Rockville, MD) for each of the 46 Mycobacterium avium ssp. paratuberculosis infectious dairy cows. Subplots are labelled by the blinded identification number for each cow. Note that cycle threshold values equal to or greater than 42 are considered negative according to the kit instructions $(\mathrm{ND}=$ not detected). 
Table 2. Total sensitivity (\%; total positive samples), along with shedding-level stratifications (\%), of fecal solid culture (Herrold's egg yolk medium with mycobactin J), fecal broth culture (Trek ESP system; Thermo Scientific, Oakwood Village, OH), fecal real-time PCR (qPCR; VetAlert, Tetracore, Rockville, MD), and milk ELISA (Paracheck, Prionics, Switzerland) recorded for concurrent samples collected monthly for 12 mo from 46 Mycobacterium avium ssp. paratuberculosis infectious cows from 7 herds

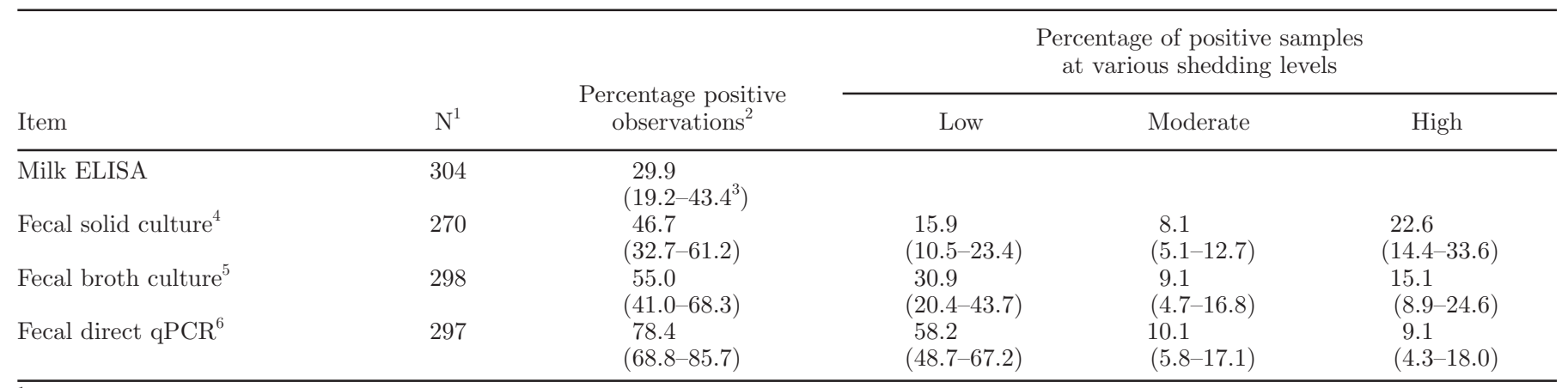

${ }^{1}$ Total number of observations.

${ }^{2}$ Sensitivity regardless of shedding levels.

${ }^{3} 95 \%$ confidence interval, adjusting for clustering by cow.

${ }^{4}$ Shedding categories determined with solid culture: low ( $<10 \mathrm{cfu}$ per culture tube), moderate (10 to $\left.50 \mathrm{cfu}\right)$, and high ( $>50 \mathrm{cfu}$; Crossley et al., 2005).

${ }^{5}$ Shedding categories determined with broth culture: low ( $>28 \mathrm{~d}$ to a positive signal), moderate (21 to $\left.28 \mathrm{~d}\right)$, and high $(<21 \mathrm{~d}$; Shin et al., 2000 , 2001).

${ }^{6}$ Shedding categories determined with real-time PCR: low (30 to $42 \mathrm{Ct}$ ), moderate $(26-30 \mathrm{Ct}$ ), and high (<26 Ct; Laurin et al., 2015).

to the threshold were more likely to be positive at a repeat sampling, with increased Se of milk ELISA at a 12 -mo repeat test than only at 6 mo. Due to the sparseness of the observations (Figure 1), the effect of stage of lactation could not be separated from the effect of season, and therefore the higher scores predicted during winter months is notable and will require a larger study to investigate this finding. A recent longitudinal study of the effect of DIM by Eisenberg et al. (2015) found that milk yield was a significant determinant of ELISA sample-to-positive ratios, with recommenda- tions to sample either early or late lactation cows, with preference to early lactation for management ease, for cows over first parity. Other studies also suggested that milk antibody concentration may be greater both in early (first or second weeks) and late (greater than $45 \mathrm{wk}$ ) lactation than in the third to twelfth weeks of lactation (Nielsen et al., 2002; Lombard et al., 2006) due to colostral antibody levels, milk dilution effects, and Johne's disease stage (Nielsen and Toft, 2012). As mentioned earlier, our study found that late lactation milk MAP antibody increase could not be separated

Table 3. Proportion (\%) of positive milk ELISA results (Parachek, Prionics, Switzerland) stratified within each shedding category for fecal solid culture (Herrold's egg yolk medium with mycobactin J), fecal broth culture (Trek ESP system, Thermo Scientific, Oakwood Village, OH), and fecal real-time PCR (qPCR; VetAlert, Tetracore, Rockville, MD) for concurrent samples collected monthly over 12 mo from 46 Mycobacterium avium ssp. paratuberculosis infectious cows from 7 herds

Shedding level

\begin{tabular}{|c|c|c|c|c|c|}
\hline \multirow[b]{2}{*}{ Item } & \multirow[b]{2}{*}{$\mathrm{N}^{1}$} & \\
\hline & & Negative & Low & Moderate & High \\
\hline Fecal broth culture ${ }^{4}$ & 298 & $\begin{array}{l}3.7(134) \\
{[1.6-8.3]^{\mathrm{a}}}\end{array}$ & $\begin{array}{l}43.5(92) \\
{[25.3-63.6]^{\mathrm{a}}}\end{array}$ & $\begin{array}{c}51.8(27) \\
{[22.1-80.3]^{\mathrm{a}}}\end{array}$ & $\begin{array}{l}68.9(45) \\
{[49.0-83.6]^{\mathrm{a}}}\end{array}$ \\
\hline Fecal direct $\mathrm{qPCR}^{5}$ & 297 & $\begin{array}{c}{[1.0-0.3]} \\
3.1(67) \\
{[0.7-13.0]^{\mathrm{a}}}\end{array}$ & $\begin{array}{c}24.3(173) \\
{[13.6-39.4]^{\mathrm{ab}}}\end{array}$ & $\begin{array}{c}73.3(30) \\
{[43.4-90.8]^{\mathrm{bc}}}\end{array}$ & $\begin{array}{l}{[49.0-83.0]} \\
85.2(27) \\
{[55.9-96.3]^{\mathrm{c}}}\end{array}$ \\
\hline
\end{tabular}

\footnotetext{
${ }^{\mathrm{a}-\mathrm{c}}$ Significant differences between shedding levels per fecal assay are represented by different superscript letters $(P<0.05)$.

${ }^{1}$ Total number of observations.

${ }^{2}$ Shedding categories determined with solid culture: low ( $<10 \mathrm{cfu}$ per culture tube), moderate (10 to $50 \mathrm{cfu}$ ), and high ( $>50 \mathrm{cfu}$; Crossley et al., 2005).

${ }^{3} 95 \%$ confidence interval, adjusted for clustering by cow.

${ }^{4}$ Shedding categories determined with broth culture: low ( $>28$ d to a positive signal), moderate (21 to $\left.28 \mathrm{~d}\right)$, and high ( $<21 \mathrm{~d}$; Shin et al., 2000 , 2001).

${ }^{5}$ Shedding categories determined with real-time PCR: low (30 to $42 \mathrm{Ct}$ ), moderate $(26-30 \mathrm{Ct}$ ), and high (<26 Ct; Laurin et al., 2015).
} 


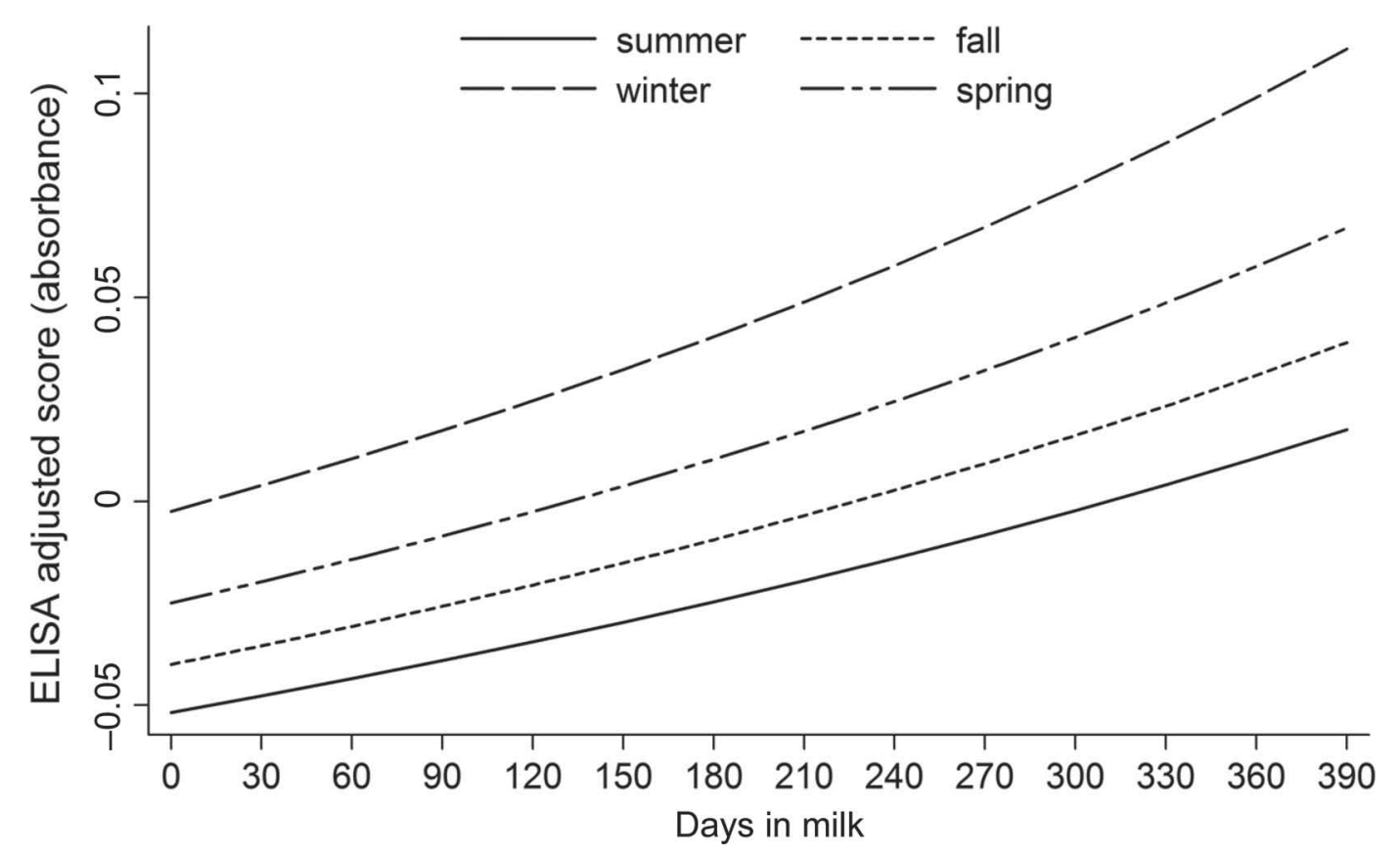

Figure 5. Predicted marginal adjusted scores for milk ELISA (Parachek, Prionics, Schlieren-Zürich, Switzerland) across lactation months within each season for milk samples collected monthly over 12 mo from 46 Mycobacterium avium ssp. paratuberculosis infectious cows from 7 herds. Marginal scores were calculated on the mixed linear model with farm and cow level random effects and mean age (4.5 yr).

from season and was greater during winter months. Recently, Cazer et al. (2013) assessed seasonal effects on bulk tank milk ELISA results and detected an increase in bulk tank MAP antibodies during summer and a decrease during winter in North America (New York, Wisconsin, and Oregon). They suggest that the seasonal effect found in their study may be related to seasonal changes in MAP bacterial load and may be dependent upon seasonal calvings, higher herd prevalence, or a few high shedding cows. Collins et al. (2005) also pointed out that a humoral immunity peak can correspond to increased exposure to MAP during specific seasons. Further study in our region is required to assess potential causes that could lead to the observed increase in MAP ELISA scores in individual cow milk samples in winter.

\section{CONCLUSIONS}

Accurate knowledge of shedding and diagnostic patterns is important for reducing MAP transmission risks and for development of improved diagnostic and screening protocols. Our study assessed the relative Se of a milk ELISA for MAP-infectious cows and investigated the seasonal and lactational patterns on MAP antibody concentrations in milk. Our data indicated relative milk ELISA Se of 30\%, with increasing ELISA
Se in relation to increased fecal shedding and increased age. Notably, a corresponding effect for season (winter versus summer) and lactation stage (increasing DIM) on ELISA score values indicates variation over time and highlights areas that require further study in the understanding of this disease.

\section{ACKNOWLEDGMENTS}

Funding for this research and personal support was provided by Dairy Farmers of Canada (Ottawa, Ontario, Canada), National Sciences and Engineering Research Council (Ottawa, Ontario, Canada), Innovation PEI (Charlottetown, Prince Edward Island, Canada), and Maritime Quality Milk (AVC, Charlottetown, Prince Edward Island, Canada). Funding sources were grant-based only, and the funders had no role in study design, data collection and analysis, decision to publish, or preparation of the manuscript. The authors thank the Farm Services and Maritime Quality Milk programs at AVC for assistance with sample collection and laboratory processing, especially Maria Vasquez, Natasha Robinson, and Jane Saunders. The authors also acknowledge the participating dairy producers. The authors thank William Chalmers (AVC) for technical assistance in preparation of the manuscript and Henrik Stryhn (AVC) for statistical advice. 


\section{REFERENCES}

Carpenter, T. E., I. A. Gardner, M. T. Collins, and R. H. Whitlock. 2004. Effects of prevalence and testing by enzyme-linked immunosorbent assay and fecal culture on the risk of introduction of $M y$ cobacterium avium ssp. paratuberculosis-infected cows into dairy herds. J. Vet. Diagn. Invest. 16:31-38.

Cazer, C. L., R. M. Mitchell, K. M. Cicconi-Hogan, M. Gamroth, R. M. Richert, P. L. Ruegg, and Y. H. Schukken. 2013. Associations between Mycobacterium avium ssp. paratuberculosis antibodies in bulk tank milk, season of sampling and protocols for managing infected cows. BMC Vet. Res. 9:234.

Clark, D. L. M. Jr., J. J. Koziczkowski, R. P. Radcliff, R. A. Carlson, and J. L. E. Ellingson. 2008. Detection of Mycobacterium avium subspecies paratuberculosis: Comparing fecal culture versus serum enzyme-linked immunosorbent assay and direct fecal polymerase chain reaction. J. Dairy Sci. 91:2620-2627.

Collins, M. T., S. J. Wells, K. R. Petrini, J. E. Collins, R. D. Schultz, and R. H. Whitlock. 2005. Evaluation of five antibody detection tests for diagnosis of bovine partuberculosis. Clin. Diagn. Lab. Immunol. 12:685-692.

Crossley, B. M., F. J. Zagmutt-Vergara, T. L. Fyock, R. H. Whitlock, and I. A. Gardner. 2005. Fecal shedding of Mycobacterium avium ssp. paratuberculosis by dairy cows. Vet. Microbiol. 107:257-263.

Dohoo, I. R., S. W. Martin, and H. Stryhn. 2009. Veterinary Epidemiologic Research. 2nd ed. VER Inc., Charlottetown, PE, Canada

Donat, K., K. Schlotter, G. Erhardt, and H. R. Brandt. 2014. Prevalence of paratuberculosis in cattle and control measures within the herd influence the performance of ELISA tests. Vet. Rec. 174:119. https://doi.org/10.1136/vr.101533.

Eisenberg, S. W. F., E. Veldman, V. P. M. G. Rutten, and A. P. Koets. 2015. A longitudinal study of factors influencing the result of a Mycobacterium avium ssp. paratuberculosis antibody ELISA in milk of dairy cows. J. Dairy Sci. 98:2345-2355. https://doi. org/10.3168/jds.2014-8380.

Garry, F. 2011. Control of paratuberculosis in dairy herds. Vet. Clin. North Am. Food Anim. Pract. 27:599-607.

Hendrick, S. H., T. E. Duffield, D. E. Kelton, K. E. Leslie, K. D. Lissemore, and M. Archambault. 2005. Evaluation of enzyme-linked immunosorbent assays performed on milk and serum samples for detection of paratuberculosis in lactating dairy cows. J. Am. Vet. Med. Assoc. 226:424-428.

Huda, A., G. Jungersen, and P. Lind. 2004. Longitudinal study of interferon-gamma, serum antibody and milk antibody responses in cattle infected with Mycobacterium avium ssp. paratuberculosis. Vet. Microbiol. 104:43-53.

Laurin, E. L., M. Chaffer, J. T. McClure, S. L. B. McKenna, and G. P. Keefe. 2015. The association of detection method, season, and lactation stage on identification of fecal shedding in Mycobacterium avium ssp. paratuberculosis infectious dairy cows. J. Dairy Sci. 98:211-220. https://doi.org/10.3168/jds.2014-8406.

Lavers, C. J., H. W. Barkema, I. R. Dohoo, S. L. B. McKenna, and G. P. Keefe. 2014. Evaluation of milk ELISA for detection of $M y$ cobacterium avium subspecies paratuberculosis in dairy herds and association with within-herd prevalence. J. Dairy Sci. 97:299-309.

Lavers, C. J., I. R. Dohoo, S. L. B. McKenna, and G. P. Keefe. 2015. Sensitivity and specificity of repeated test results from a com- mercial milk enzyme-linked immunosorbent assay for detection of Mycobacterium avium subspecies paratuberculosis in dairy cattle. J. Am. Vet. Med. Assoc. 246:236-244.

Lavers, C. J., S. L. B. McKenna, I. R. Dohoo, H. W. Barkema, and G. P. Keefe. 2013. Evaluation of environmental fecal culture for $M y-$ cobacterium avium subspecies paratuberculosis detection in dairy herds and association with apparent within-herd prevalence. Can. Vet. J. 54:1053-1060.

Lombard, J. E. T. M. Byrem, B. A. Wagner, and B. J. McCluskey. 2006. Comparison of milk and serum enzyme linked immunosorbent assays for diagnosis of Mycobacterium avium subspecies paratuberculosis infection in dairy cattle. J. Vet. Diagn. Invest. 18:448-458.

Nielsen, S. S. 2008. Transitions in diagnostic tests used for detection of Mycobacterium avium ssp. paratuberculosis infections in cattle. Vet. Microbiol. 132:274-282.

Nielsen, S. S., C. Enevoldsen, and Y. T. Gröhn. 2002. The Mycobacterium avium ssp. paratuberculosis ELISA response by parity and stage of lactation. Prev. Vet. Med. 54:1-10.

Nielsen, S. S. and A. K. Ersbøll. 2006. Age at occurrence of Mycobacterium avium subspecies paratuberculosis in naturally infected dairy cows. J. Dairy Sci. 89:4557-4566.

Nielsen, S. S., and N. Toft. 2006. Age-specific characteristics of ELISA and fecal culture for purpose-specific testing for paratuberculosis. J. Dairy Sci. 89:569-579.

Nielsen, S. S., and N. Toft. 2008. Ante mortem diagnosis of paratuberculosis: A review of accuracies of ELISA, interferon- $\gamma$ assay and faecal culture techniques. Vet. Microbiol. 129:217-235.

Nielsen, S. S., and N. Toft. 2012. Effect of days in milk and milk yield on testing positive in milk antibody ELISA to Mycobacterium avium ssp. paratuberculosis in dairy cattle. Vet. Immunol. Immunopathol. 149:6-10.

Nielsen, S. S., N. Toft, and H. Okura. 2013. Dynamics of specific anti-Mycobacterium avium ssp. paratuberculosis antibody response through age. PLoS One 8:e63009. https://doi.org/10.1371/journal. pone.0063009.

Shin, S. J., S. G. Kim, L. J. Miller, P. R. Harpending, and D. H. Lien. 2000. A new liquid culture method, the Trek ESP Culture System II, for the rapid detection of Mycobacterium avium ssp. paratuberculosis in bovine clinical samples. Page 29 in Proc. Am. Assoc. Vet. Lab. Diagnosticians, Birmingham, AL. American Association of Veterinary Laboratory Diagnosticians, Visalia, CA

Shin, S. J., S. G. Kim, L. J. Miller, P. R. Harpending, V. H. Patten, S. M. Stehman, C. A. Rossiter, P. L. McDonough, and D. H. Lien 2001. Further evaluation of ESP Culture System II for detection of Mycobacterium avium ssp. paratuberculosis in bovine fecal samples. Page 39 in Proc. Am. Assoc. Vet. Lab. Diagnosticians, Hershey, PA. American Association of Veterinary Laboratory Diagnosticians, Visalia, CA.

Tiwari, A., J. A. VanLeeuven, S. L. McKenna, G. P. Keefe, and H. W. Barkema. 2006. Johne's disease in Canada. Part 1: Clinical symptoms, pathophysiology, diagnosis and prevalence in dairy herds. Can. Vet. J. 47:874-882

Toft, N., S. S. Nielsen, and E. Jorgensen. 2005. Continuous data diagnostic tests for paratuberculosis as a multistage disease. J. Dairy Sci. 88:3923-3931. 